\title{
A microfluidic device towards shear stress analysis of clonal expanded endothelial cells
}

\author{
Emilie WEIBULL*, Shunsuke MATSUI**, Helene ANDERSSON SVAHN* and Toshiro OHASHI*** \\ *Division of Proteomics and Nanobiotechnology, Science for Life Laboratory, KTH-Royal Institute of Technology \\ 17165 Solna, Sweden \\ E-mail: helene.andersson.svahn@scilifelab.se \\ ${ }^{* *}$ Graduate School of Engineering, Hokkaido University \\ 060-8628 Sapporo, Hokkaido, Japan \\ *** Faculty of Engineering, Hokkaido University \\ 060-8628 Sapporo, Hokkaido, Japan
}

Received 28 November 2013

\begin{abstract}
The endothelial cells lining our cardiovascular system are constantly affected by shear stress, which can alter both the morphology and biological activity of the cells. Methods to study the basic shear stress response by creating stable flow profiles on the macro scale are well established, but they do not allow the generation of controlled high precision flow profiles. The emergence of microfluidic devices has enabled well-defined individual cellular response studies on endothelial cells in scale-relevant tools. However, so far, no shear stress studies on clonal heterogeneity have been published. We have developed a novel bioassay system to study several shear stress conditions in parallel on clonal expanded single cells. The device consists of a silicon/glass microwell slide with integrated polydimethylsiloxane microchannels, which delivers shear stress to cells in a well-controlled manner using micropumps. The flow behavior of the device was numerically characterized by computational fluid dynamics analysis, which confirmed that the desired fluid-imposed shear stress was obtained. Bovine aortic endothelial cells were cultured in the microwells for 24 hours and then subjected to a fluid shear stress of up to $2.0 \mathrm{~Pa}$ for 6 hours. The results showed that alignment and elongation of the endothelial cells along the flow direction were dependent on the level of shear stress applied. It was demonstrated that multiple experimental conditions can be examined simultaneously within a single device and the compartmentalized structure of the microwell slide can be used to ensure physical separation of cells in individual wells. Moreover, it was shown that the device could reduce consumption of expensive reagents and enable screening of rare samples.
\end{abstract}

Keywords: Endothelial cells, Bioassay system, MEMS, Microfluidics, Fluid shear stress, Microwells

\section{Introduction}

Endothelial cells (EC) lining the blood vessels and other cardiovascular tissue in our circulatory system are continuously affected by mechanical forces. Shear stress is known to affect both the morphology and biological activity of ECs in their local microenvironment (Young and Simmons, 2010), e.g., leading to changes in endothelial permeability (Kwei, et al., 2004), blood formation (Eilken, et al., 2009), vasoregulation (Barakat, 1999) and arterial remodeling (Langille and O'Donnell, 1986). Furthermore, vascular diseases such as atherosclerosis are primarily localized to regions with abnormal shear stress patterns (van der Meer, et al., 2010). Fluid flow systems for studying basic endothelial biology on the macro scale have been available for over twenty years and are well established. Many of these systems are capable of producing well-defined fluid flows for biologically relevant shear stresses. However, systems on the macro scale are difficult to regulate and do not allow the generation of high precision fluid profiles, due to higher Reynolds numbers. In addition, they lack the capability for tracking individual or clonal expanded cells. Other drawbacks include their large consumption of reagents, contamination risks and limited portability and throughput (Song, et al., 2005).

Highly versatile microfluidic systems have recently been developed capable of investigating shear stress-dependent cellular reactions that are not feasible to analyze using macro scale systems. There are many advantages to using 
miniaturized devices. Firstly, microfluidic channel dimensions are physically similar to those of the smaller vessels in the cardiovascular system, suggesting that micro scale devices are better suited for mimicking in vivo conditions. Secondly, microfluidic channels typically have low Reynolds number, thus exhibiting laminar flow, which enables a highly controlled fluidic profile. Thirdly, the miniaturized format makes it possible to follow individual cells, e.g., clones expanded from single cells, and perform controlled time-lapse studies (Song, et al., 2005). Additionally, devices on the micro scale enable high-throughput analysis, allowing thousands of individual cells to be screened under multiple conditions simultaneously. Microfluidic-based devices also require smaller volumes of reagents, which could reduce the cost of the assay and save precious samples. For example, a microfluidic device proposed by Chen et al. only consumes a volume of 2-3 $\mu$ ( Chen, et al, 2013), whereas a typical macro system would consume tens of milliliters. Microfluidic systems also decrease the risk of contaminants because they provide a closed environment, in contrast to open macro systems.

There have been several recent publications exhibiting alignment of ECs in microfluidic channels (van der Meer, et al., 2010; Song, et al., 2005; Chen, et al., 2013; Sasaki, et al., 2012 and Rossi, et al., 2009). Furthermore, some researchers have attempted to simulate the pulsatile and oscillatory shear stress that ECs experience in vivo (Chen, et al, 2013 and Shao, et al., 2009). However, to date, only a few examples of microfluidic devices that allow screening of several shear stress conditions simultaneously have been published (Song, et al., 2005; Rossi, et al., 2009; Lu, et al., 2004 and Conant, et al., 2011), and none of those designs have multiple isolated culture chambers for each condition, and can therefore not be used for clonal heterogeneity analysis.

We have previously designed a microwell slide in the format of a traditional microscopic slide that contains 672 wells $(14 \mathrm{x} 48)$. The walls of the wells are tampered, facilitating liquid exchange, and each well can hold up to $500 \mathrm{nl}$. Previously, we have shown that the slide can be successfully used for single cell and clonal heterogeneity studies (Lindström, et al., 2008 and Lindström, et al., 2009a), and be combined with integrated microfluidic channels and pumps (Lindström, et al., 2009b).

In the present study, we have further developed the microwell slide with four integrated microfluidic channels to analyze shear stress dependent responses of ECs. The device was developed and validated as a first step in creating a device able to screen for clone heterogeneity derived from single cells. This is the first time that a device with multiple shear stress conditions has been developed in combination with the possibility of isolating single clones. There are several advantages compared to other fluid-imposed devices, i) it enables future shear stress heterogeneity studies on cells derived from single cells for ii) 48 wells in each channel, which are affected by the same shear stress, iii) multiple shear stress conditions, iv) standardized dimensions, can be analyzed in traditional instruments, v) a thin glass bottom enabling high-resolution imaging $(175 \mu \mathrm{m})$.

The flow behavior in the device was numerically characterized by computational fluid dynamics analysis (CFD), which confirmed that the desired fluid-imposed shear stress was obtained under the different conditions tested $(0.1,0.5$, 1.0 and 2.0 Pa). The shear stress was applied for 6 hours to confluent bovine aortic endothelial (BAE) cells cultured in the microwells. The cell orientation was assessed optically, showing that up to $40-50 \%$ of the ECs aligned to the direction of the flow. The percentage of aligned cells increased with increasing shear stress. The next step will be to study the clonal heterogeneity of ECs at the single cell level and discuss application in studies of cardiovascular diseases. To the best of our knowledge, no previous publications have examined the effect of shear stress on individual ECs after clonal expansion.

\section{Materials and Methods}

\subsection{Endothelial cell culture}

BAE cells were purchased from Cell Applications (San Diego, CA, USA) and cultured in a tissue culture flask (T-175, Becton, Dickinson and Company, NJ, USA) in Dulbecco's modified Eagle medium (DMEM, Invitrogen, NY, USA) supplemented with $10 \%$ heat-inactivated fetal bovine serum (FBS, Invitrogen) and 100 units/ml of penicillin-streptomycin (Invitrogen). The BAE cells were cultured at $37^{\circ} \mathrm{C}$ in an incubator with $5 \% \mathrm{CO} 2 / 20 \% \mathrm{O} 2 / 75 \%$ $\mathrm{N} 2$ until confluent (after 4-5 days) and then passaged at a 1:3 or 1:4 split ratio in the flask using $0.5 \%$ trypsin-EDTA (Invitrogen). Cells from passages 4 - 8 were used in the shear stress experiments. 


\subsection{Microwell slide-based flow-imposed device}

A schematic of the shear stress-imposed device is shown in Fig. 1(a). The device was comprised out of two parts: a microwell slide and microfluidic channel system fabricated in polydimethylsiloxane (PDMS). The microwell slide has been described previously (Lindström, et al., 2009a, 2009b) and consisted of a $500 \mu \mathrm{m}$ thick, $75 \mathrm{~mm}$ x $25 \mathrm{~mm}$ etched silicon grid with an array of 14 x 48 microwells. The silicon grid was bonded to a $175 \mu \mathrm{m}$ thin glass slide to enable high-content imaging. The integrated device, i.e., microchannels and microwell slide, is shown in Fig. 1(b). The configuration of the microchannel and microwell interface is shown in Fig. 1(c). Four parallel microchannels were fabricated in the device, enabling different degrees of fluid shear stress to be applied simultaneously, as shown in Fig. 1 (d). For creating the microchannel layer, a reusable master was designed using 3D-CAD software and then the mask pattern was transferred to laser lithography apparatus (Eden2600V, Fasotec, Chiba, Japan). A concave array structure was fabricated by laser lithography using an ultra-violet curable resin. The surface of the master was coated with trichlorosilane to prevent PDMS adhesion. Next, soft lithography casting was performed to produce PDMS replicas (1:10 mixture) of the concave array structure, creating the microchannel layer. A cured 5 mm-thick PDMS sheet with 4

A

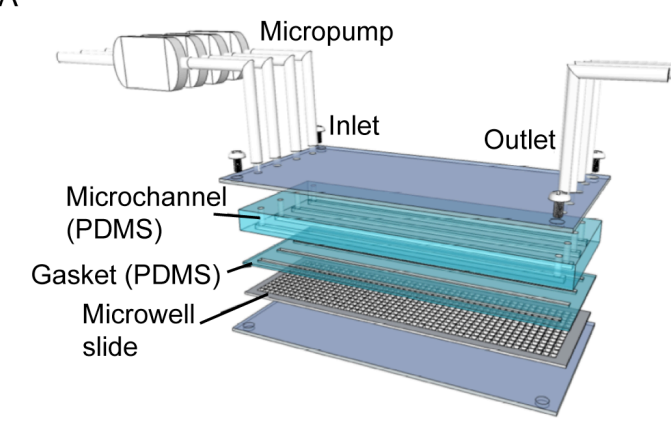

C

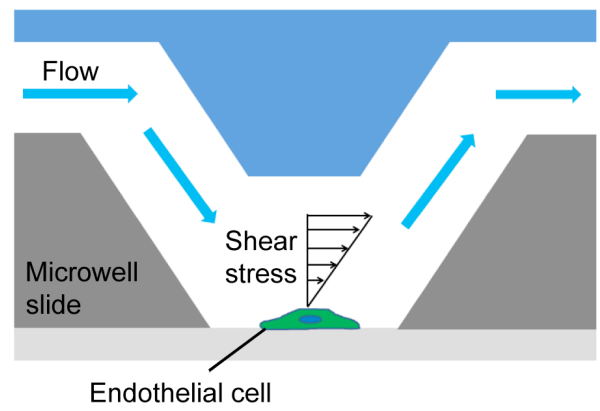

B

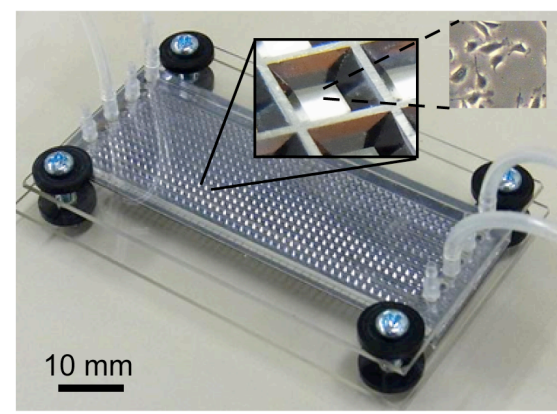

D

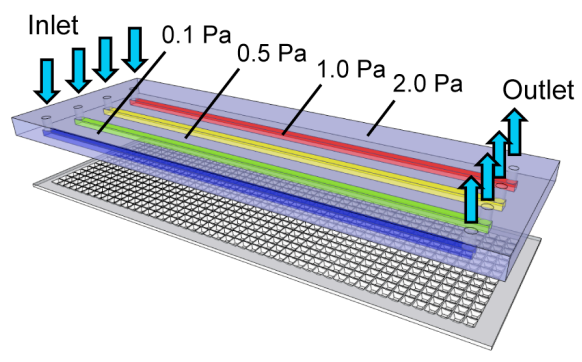

Fig. 1 (a) Schematic of the flow-imposed device composed of a PDMS layer with four microchannels, a PDMS gasket layer and a silicon microwell slide. (b) Photograph of the device and an example of cell culture in the microwells. (c) Configuration of the microchannel/microwell interface. (d) Schematic of the device with application of four different shear stresses $(0.1,0.5,1.0$ and $2.0 \mathrm{~Pa})$.

parallel microchannels $1340 \mu \mathrm{m}$ wide x $100 \mu \mathrm{m}$ deep (the gasket layer) was fabricated separately. Inlet and outlet holes with a diameter of $1.5 \mathrm{~mm}$ were made on both sides of the microchannels. The PDMS microchannel layer, the PDMS gasket layer and the microwell slide were bonded together using a plasma bonding technique and tightly fixed with glass plates and nuts/bolts, as shown in Fig. 1(b). Four piezoelectric diaphragm micropumps (mp-6, Bartels, Germany) were used to dispense cell culture medium onto a confluent cell layer via $1.5 \mathrm{~mm}$ inner diameter silicone tubing. Flow rates were coordinated to deliver shear stresses of 0.1, 0.5, 1.0 and 2.0 Pa using LabVIEW software based on the results of finite element calculation.

\subsection{CFD analysis}

Flow conditions in the microchannels were analyzed by using computational fluid dynamics (CFD) analysis (ANSYS Ver. 12, Cybernet, Tokyo, Japan). The magnitude of the fluid-imposed shear stress on BAE cells cultured in the microwells was estimated using a finite element model constructed with 147889 tetrahedral elements (Fig. 2 (a)). The boundaries at the top, bottom and sidewalls of the simulated domain were set to non-slip walls and the outlet was 
set to $0 \mathrm{~Pa}$. The flow was assumed to be laminar, steady state, and viscous. The density of the elements was set at 1 $\mathrm{g} / \mathrm{cm}^{3}$ and the viscosity was set at $9.6 \times 10^{-4} \mathrm{~Pa} \cdot \mathrm{s}$, which is similar to that of the cell culture medium. Finally, flow velocities at the inlets were determined in order to impose the desired fluid shear stress on the confluent cultured BAE cells.

A

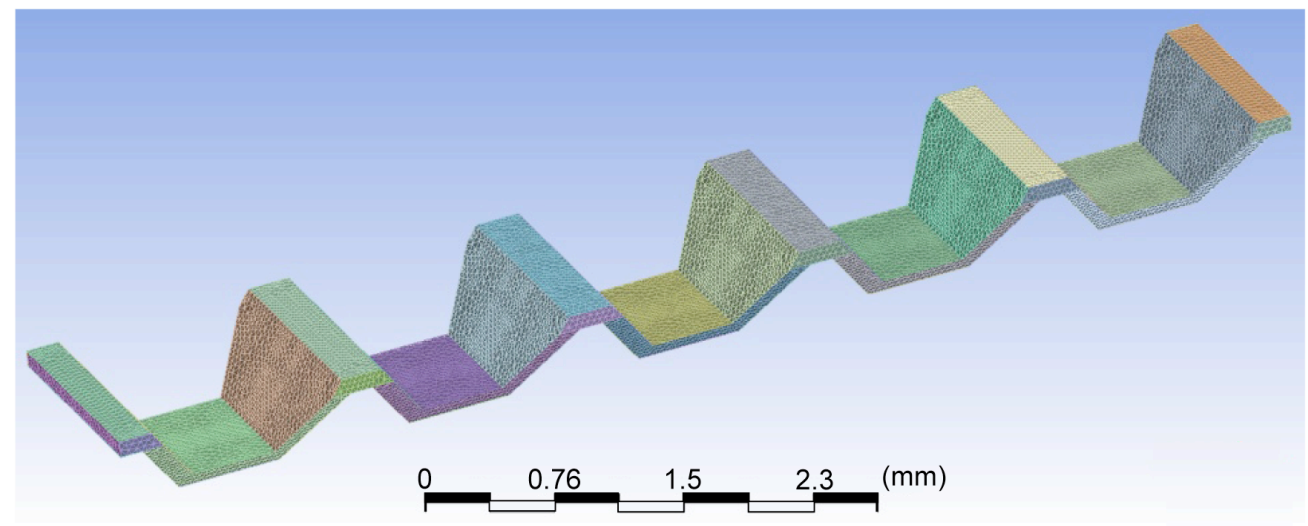

B Flow

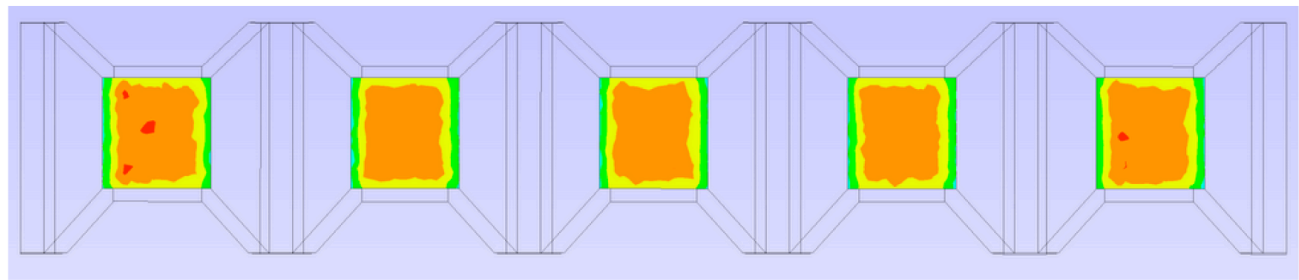

Fig. 2 (a) Finite element model of the microchannel. The displayed color is automatically generated by the software and holds no purpose. (b) CFD analysis showing the shear stress distribution at the bottom of the wells (2.0 Pa shear stress).

\subsection{Flow-imposed experiments}

Before seeding the cells, the microwell slide was coated with $50 \mu \mathrm{g} / \mathrm{ml}$ fibronectin (Sigma-Aldrich Japan, Tokyo, Japan). The BAE cells were seeded at a density of $4.0 \times 10^{5}$ cells $/ \mathrm{ml}$ and cultured in the microwells for $24 \mathrm{~h}$, after which the cells were exposed to the above-mentioned fluid shear stress for 6 hours.

\subsection{Optical analysis}

Cells in the microwell-structured microchannels were monitored before and after conducting the flow-imposed experiment using an inverted fluorescence microscope (IX81, Olympus, Tokyo, Japan). The angle of the cell orientation (Levesque and Nerem, 1985) was evaluated using public domain software (Image J 1.45g, National Institutes of Health, MD, USA). The cell orientation was defined as the deviation from the major axis of an equivalent ellipse relative to the direction of the flow, giving a cell orientation ranging from $0^{\circ}$ to $180^{\circ}$.

\section{Results}

The new device, consisting of a silicon/glass microwell slide with integrated PDMS microchannels for delivering shear stress to cells in a well-controlled manner using micropumps, was designed, fabricated and evaluated. The flow behavior in the device was numerically characterized by CFD analysis, which confirmed that the desired fluid-imposed shear stress was obtained. The flow rates at the inlets were determined relative to shear stresses of $0.1,0.5,1.0$ and 2.0 $\mathrm{Pa}$, resulting in $0.013 \mathrm{ml} / \mathrm{min}, 0.06 \mathrm{ml} / \mathrm{min}, 0.12 \mathrm{ml} / \mathrm{min}$ and $0.2 \mathrm{ml} / \mathrm{min}$, respectively. The fluid-imposed shear stress generated on the bottom of the microwells are shown for a shear stress of $2 \mathrm{~Pa}$ in Fig. 2 (b). A uniform channel flow was clearly observed in the microchannel without indication of countercurrent flow. The bottom of the microwells was mainly exposed to shear stresses ranging from 1.6 $\mathrm{Pa}$ to $2.0 \mathrm{~Pa}$. The experimental shear stress experiments were therefore designed according to the theoretically calculated results. 
The biocompatibility of the developed device was evaluated by culturing BAE cells. The cells were seeded using limited dilution, which resulted in approximately 50 cells/well. The cells morphology and ability to proliferate was monitored and evaluated for a few days. There was no indication that the device altered the cells' behavior, and therefore the device was considered to be biocompatible.

Fluid-imposed shear stresses of 0.1, 0.5, 1.0 and 2.0 Pa were applied in parallel to the four different channels on the device. Around $100 \mathrm{BAE}$ cells/well were seeded using limited dilution and cultured for $24 \mathrm{~h}$, until confluent, prior to exposure to the fluid-imposed shear stress. The BAE cells growing in the microwells were exposed to the different shear stresses during 6 hours after which they were optically evaluated. Fig. 3 (a) shows typical bright-field images of the BAE cells before and after application of 2.0 Pa fluid-imposed shear stress. The angle of the cell orientation was analyzed using image analysis, as shown in Fig. 3 (b) for $2.0 \mathrm{~Pa}$ and is based on around 100 cells. The percentage of cells aligned along the direction of flow increased with increasing shear stress. The cells in the wells with the highest shear stress displayed an elongated morphology along the direction of flow after the experiments. Due to the small size of the wells it was feasible to evaluate single cells in the wells. For $0.1 \mathrm{~Pa}$, no cell alignment to the direction of the flow was observed, whereas for the higher shear stresses of 1.0 and $2.0 \mathrm{~Pa}$, the percentage of cells aligned along the direction of flow (Fig. 3 (c)) $\left( \pm 0^{\circ}-30^{\circ}\right.$ and $\left.\pm 150^{\circ}-180^{\circ}\right)$ clearly increased. Fig. 4 shows the percentage of cells aligned to the direction of flow for the four different shear stresses used. No inter-well differences in the cell orientation were detected, as expected since the starting material was a mixed population of cells in all wells. This confirms that the location of the well within a channel does not affect the morphology of the fluid-imposed cells.

A

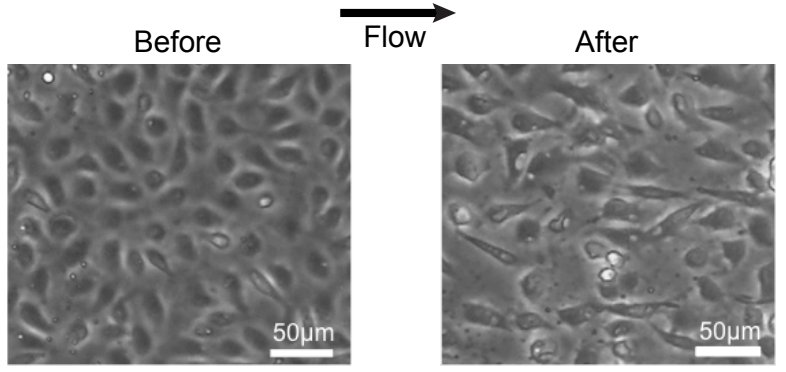

B

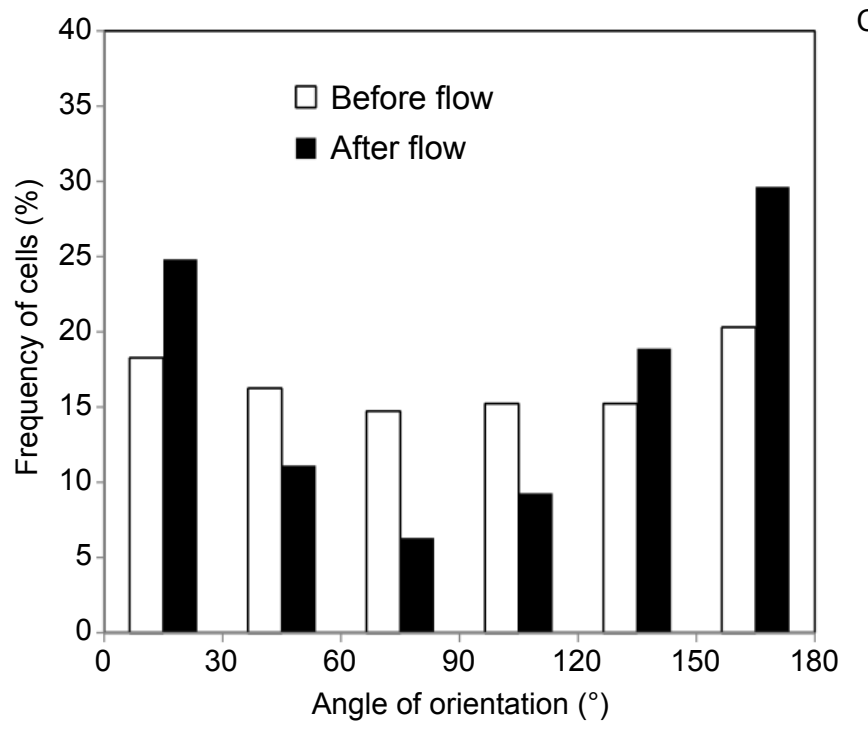

C

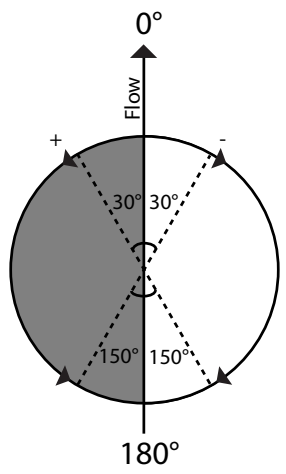

Fig. 3 (a) Bright-field images of BAE cells before and after experiments (the flow direction is from left to right), and (b) frequency of cells versus the angle of cell orientation before and after application of 2.0 Pa fluid shear stress for 6 hours. (c) A Schematic diagram illustrating the used angles. 


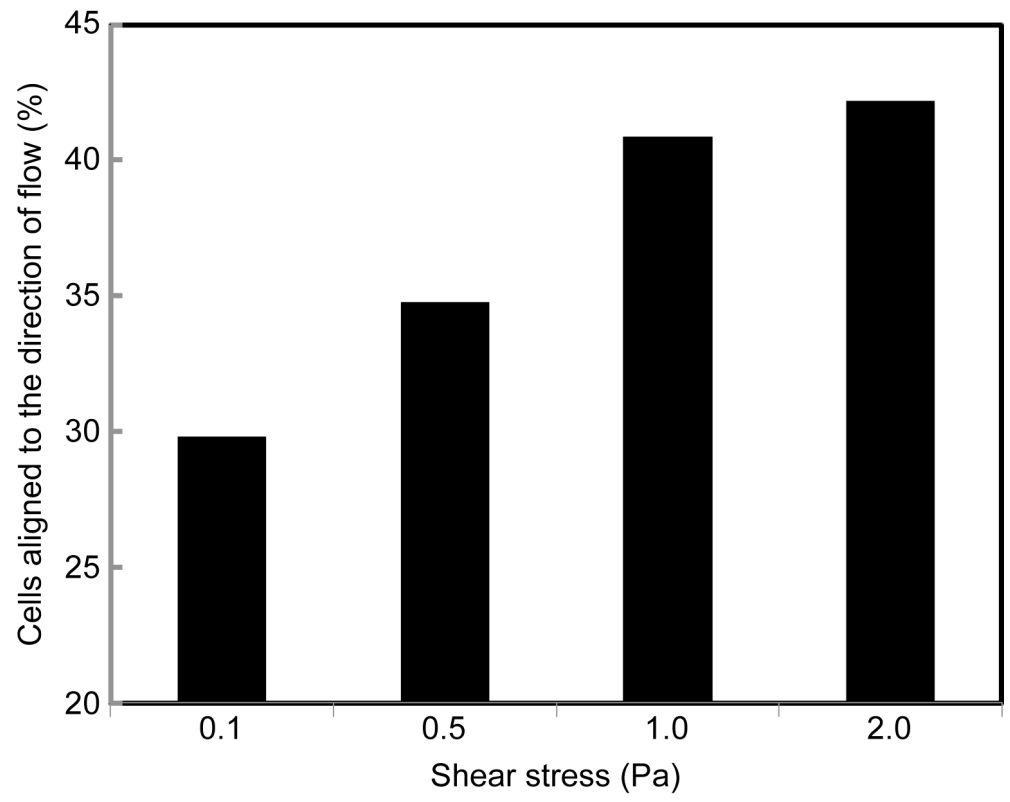

Fig. 4 Percentage of BAE cells aligned to the direction of flow for the four different of shear stresses tested ( 0.1 , $0.5,1.0$ and $2.0 \mathrm{~Pa})$.

\section{Discussion}

We developed a microfluidic device that enables analysis of multiple shear stress conditions at a single cell level for clonally expanded cells. The experiments were performed under clinically relevant shear stress conditions, e.g., the average blood pressure in aortas is known to impose a shear stress of around 2.0 Pa. There was a clear change in the cell alignment and elongation of BAE cells exposed to 1.0 and 2.0 $\mathrm{Pa}$ in our device and a small increase for cells exposed to $0.5 \mathrm{~Pa}$. This is in accordance with previously reported results for endothelial cells exposed to a fluid shear stress of 2.0 Pa (Kataoka, et al., 1998 and Ohashi, et al., 2002) and over 0.8 Pa (Dewey, et al., 1981).

There are several advantages with our device. Firstly, the integrated device takes advantage of the microwells' compartmentalized structure and the controlled environment of the microfluidic channels, enabling both high spatial and temporal control. There are 48 wells in each channel, which can be regarded as separate cultivation chambers, allowing cells to be physically separated in different wells. Secondly, a conventional fluid-imposed setup usually requires changes to the layout in order to run different experimental conditions, e.g., employing either a flexible channel cross-section with a fixed flow rate or a flexible flow rate with a fixed cross-section. Our device can run four parallel shear stress conditions simultaneously, and the number of channels can be increased or decreased depending on the desired application. Thirdly, the thin glass bottom of the microwell slide enables high-content analysis and monitoring of clonal expanded single cells over time. Other advantages of the device include a reduced culture medium volume. For example, conventional bulk devices, such as a $35 \mathrm{~mm}$-diameter cell culture dish, require of the order of $\mathrm{ml}$ of medium, whereas the proposed device only requires a volume of the order of $\mu 1$. Moreover, the device can reduce the number of cells required from $10^{6}$ cells to $10^{2}$ cells, a reduction by 10000 fold. Hence, the device can reduce the cost of the assay and allow experiments on rare cells.

A limitation of the presented device is that controlled single cell seeding is not possible because cells are introduced into the device after bonding. This means that single cell seeding has to be performed using limited dilution, where cells are diluted to the desired concentration before being introduced into the device. An alternative approach would be to introduce the cells before bonding, at which point cells can be sorted using fluorescence activated cell sorting (FACS) (Lindström, et al., 2009a). The gasket layer must be attached to the microwell slide using plasma bonding before cell seeding. After the desired cell proliferation has been achieved, the remaining microchannel system can be bonded at $37^{\circ} \mathrm{C}$ using either semi-cured PDMS as glue or a "chemical glue" (Lee and Chung, 2009). 


\section{Conclusions}

We have developed a microfluidic channel system to study endothelial cells exposed to several shear stress conditions in parallel. Each channel runs through 48 microwells, where each well can be defined as a distinct culture area. The integrated system allows both culturing of cells and for fluidic-imposed shear stress studies to be performed. The next step is to use the compartmentalized structure of the device to study clonal heterogeneity. The experiment can be conducted on hundreds of wells simultaneously, where the cells in each well can be expanded from a single cell. The new device has many potential clinically relevant applications, e.g., to study the effect of high blood pressure on vascular endothelial cells with implications for atherosclerosis research.

\section{Acknowledgements}

This work was financially supported by the Grants-in-Aid for Scientific Research (B) (grant no. 24300157) from the Ministry of Education, Culture, Sports, Science and Technology (MEXT) of Japan and the strategic Japanese-Swedish cooperative program on "Multidisciplinary BIO" overseen by the Japan Science and Technology Agency (JST), the Swedish Governmental Agency for Innovation Systems (VINNOVA), and the Swedish Foundation for Strategic Research (SSF).

\section{References}

Barakat, A. I., Responsiveness of vascular endothelium to shear stress: potential role of ion channels and cellular cytoskeleton (review), International Journal of Molecular Medicine, Vol.4, No.4 (1999), pp. 323-332.

Chen, H., Cornwell, J., Zhang, H., Lim, T., Resurreccion, R., Port, T., Rosengarten, G., Nordon, R. E., Cardiac-like flow generator for long-term imaging of endothelial cell responses to circulatory pulsatile flow at microscale, Lab on a Chip, Vol.13, No.15 (2013), pp. 2999-3007.

Conant, C. G., Schwartz, M. A., Beecher J. E., Rudoff, R. C., Ionescu-Zanetti, C., Nevill, J. T., Well plate microfluidic system for investigation of dynamic platelet behavior under variable shear loads, Biotechnology and Bioengineering, Vol.108, No.12 (2011), pp. 2978-2987.

Dewey, C. F., Bussolari, S. R., Gimbrone, M. A., Davies, P. F., The dynamic response of vascular endothelial cells to fluid shear stress, Journal of Biomechanical Engineering-transactions of the ASME, Vol.103, No.3 (1981), pp. 177-186.

Eilken, H. M., Nishikawa, S - I., Schroeder, T., Continuous single-cell imaging of blood generation from haemogenic endothelium, Nature, Vol.457, No.7231 (2009), pp. 896-900.

Kataoka, N., Ujita, S., Sato, M., Effect of flow direction on the morphological responses of cultured bovine aortic endothelial cells, Medical \& Biological Engineering \& Computing, Vol.36, No.1 (1998), pp. 122-128.

Kwei, S., Stavrakis, G., Takahas, M., Taylor, G., Folkman M. J., Gimbrone, M. A., García-Cardeña, G., Early adaptive responses of the vascular wall during venous arterialization in mice, American Journal of Pathology, Vol.164, No.1 (2004), pp. 81-89.

Langille, B. L., O'Donnell, F., Reductions in arterial diameter produced by chronic decreases in blood flow are endothelium-dependent, Science, Vol.231, No.4736 (1986), pp. 405-407.

Lee, N. Y., Chung, B. H., Novel poly(dimethylsiloxane) bonding strategy via room temperature "chemical gluing", Langmuir, Vol.25, No.6 (2009), pp. 3861-3866.

Levesque, M. J., Nerem, R. M., The elongation and orientation of cultured endothelial cells in response to shear stress, Journal of Biomechanical Engineering-transactions of the ASME, Vol.107, No.4 (1985), pp. 341-347.

Lindström, S., Larsson, R., Andersson-Svahn, H., Towards high-throughput single cell/clone cultivation and analysis, Electrophoresis, Vol.29, No.6 (2008), pp. 1219-1227.

Lindström, S., Eriksson, M., Vazin, T., Sandberg, J., Lundeberg, J., Frisén, J., Andersson-Svahn, H., High-density microwell chip for culture and analysis of stem cells, PLoS ONE, Vol.4, No.9 (2009a), pp. e6997.

Lindström, S., Mori K., Ohashi, T., Andersson-Svahn, H., A microwell array device with integrated microfluidic components for enhanced single-cell analysis, Electrophoresis, Vol.30, No.24 (2009b), pp. 4166-4171.

Lu, H., Koo, L. Y., Wang, W. M., Lauffenburger, D. A., Griffith, L. G., Jensen, K. F., Microfluidic shear devices for quantitative analysis of cell adhesion, Analytical Chemistry, Vol.76, No.18 (2004), pp. 5257-5264.

Ohashi, T., Ishii, Y., Ishikawa, Y., Matsumoto, T., Sato, M., Experimental and numerical analyses of local mechanical properties measured by atomic force microscopy for sheared endothelial cells, Bio-medical Materials and 
Engineering, Vol.12, No.3 (2002), pp. 319-327.

Rossi, M., Lindken, R., Hierck, B. P., Westerweel, J., Tapered microfluidic chip for the study of biochemical and mechanical response at subcellular level of endothelial cells to shear flow, Lab on a Chip, Vol.9, No.10 (2009), pp. 1403-1411.

Sasaki, N., Shinjo, M., Hirakawa, S., Nishinaka, M., Tanaka, Y., Mawatari, K., Kitamori, T., Sato, K., A palmtop-sized microfluidic cell culture system driven by a miniaturized infusion pump, Electrophoresis, Vol.33, No.12 (2012), pp. 1729-1735.

Shao, J., Wu, L., Wu, J., Zheng, Y., Zhao, H., Jin, Q., Zhao, J., Integrated microfluidic chip for endothelial cells culture and analysis exposed to a pulsatile and oscillatory shear stress, Lab on a Chip, Vol.9, No.21 (2009), pp. 3118-3125.

Song, J. W., Gu, W., Futai N., Warner, K. A., Nor, J. E., Takayama, S., Computer-controlled microcirculatory support system for endothelial cell culture and shearing, Analytical Chemistry, Vol.77, No.13 (2005), pp. 3993-3999.

van der Meer, A. D., Poot, A. A., Feijen, J., Vermes, I., Analyzing shear stress-induced alignment of actin filaments in endothelial cells with a microfluidic assay, Biomicrofluidics, Vol.4, No.1 (2010), pp. 0111031-5.

Young, E. W. K., Simmons, C. A., Macro- and microscale fluid flow systems for endothelial cell biology, Lab on a Chip, Vol.10, No.2 (2010), pp. 143-160. 